\title{
12 Membrane Clusters of Ion Channels: Size Effects for Stochastic Resonance
}

\author{
Gerhard Schmid, Igor Goychuk, and Peter Hänggi \\ Universität Augsburg, Institut für Physik, D-86135 Augsburg, Germany
}

\begin{abstract}
By use of a stochastic generalization of the Hodgkin-Huxley model we investigate the phenomenon of Stochastic Resonance (SR) for a distribution of ion channels within a cluster of variable size. In the presence of a periodic stimulus we demonstrate intrinsic $S R$ vs. decreasing patch size, or, put differently, vs. increasing internal noise strength. SR with external noise occurs only for large cluster sizes which possess suboptimal internal noise levels. In particular, SR in biology thus seemingly is rooted in the collective properties of optimally selected ion channel assemblies. Moreover, upon investigating the signal-to-noise ratio (SNR) for sub-threshold sinusoidal driving vs. driving frequency we encounter also a stochastic resonance behavior which reflects the existence of a random internal limit cycle. The occurrence of intrinsic SR in a combination with the conventional frequency resonance may be of importance for the frequency tuning in biological signal processing.
\end{abstract}

\subsection{Introduction}

Much attention is presently given to the behavior of complex networks with the particular focus being on so termed scale-free networks, which are believed to present many complex phenomena in nature [1, 2, 3, 4, 5, 6, 7]. Such networks naturally also occur in biological settings. In this spirit we focus here on the constructive role of noise on voltage gated, globally connected assemblies of ion channels. If the distribution of such ion channels consists of at least two types, excitable behavior becomes possible which in turn rules the transduction of biological information. The transduction of signals in presence of ambient, internal noise then likely makes use of a cooperative behavior between nonlinearity and noise, known under the label of Stochastic Resonance 8].

During the last decade, the effect of Stochastic Resonance (SR) - a cooperative phenomenon wherein the addition of external noise improves the detection and transduction of signals in nonlinear systems (for comprehensive surveys and relevant further references, see in [8, 9]) - has been studied experimentally and theoretically in various biological systems [10, 11, 12, 13, 14]. For example, SR has been experimentally demonstrated within the mechanoreceptive system in crayfish [10], in the cricket cercal sensory system [11], for human tactile sensation [12, visual perception [13], and response behavior of the arterial baroreflex system of humans [14. The importance of this SR-phenomenon for sensory biology is by now well established; yet, it is presently not known to which minimal 
level of the biological organization the stochastic resonance effect can ultimately be traced down. Presumably, SR has its origin in the stochastic properties of the ion channel clusters located in a receptor cell membrane. Indeed, for an artificial model system Bezrukov and Vodyanoy have demonstrated experimentally that a finite ensemble of the alamethicin ion channels does exhibit stochastic resonance [15]. This in turn provokes the question whether a single ion channel is able to exhibit SR, or whether stochastic resonance is the result of a collective response from a finite assembly of channels.

Stochastic resonance in single, biological potassium ion channels has also been investigated both theoretically [16] and experimentally [17]. Thus far, the experimental work did not convincingly reveal SR in single voltage-sensitive ion channels versus the varying temperature. Nevertheless, the SR phenomenon versus the externally added noise can occur in single ion channels if only the parameters are within a regime where the channel is predominantly dwelled in the closed state, as demonstrated within a theoretical modeling for a Shaker potassium channel [16. The manifestation of SR on the single-molecular level, is not only of academic interest, but is also relevant for potential nano-technological applications, such as the design of single-molecular bio-sensors. The origin and biological relevance of SR in single ion channels, however, remains still open.

Indeed, biological SR is a manifestation of collective properties of large assemblies of ion channels of different sorts. To display the phenomenon of excitability these assemblies must contain an assemblage of ion channels of at least two different sorts - such as, e.g., potassium and sodium channels. The corresponding mean-field model has been put forward by Hodgkin and Huxley as early as in 1952 [18] by neglecting the intrinsic fluctuations which originate from the stochastic opening and closing of channels. SR due to external noise in this primary model and related models of excitable dynamics has extensively been addressed [19, 20]. A challenge though still remains: does internal noise play a constructive role for SR? Internal noise is produced by fluctuation of the number of open channels within the assembly, and diminishes with increasing number of channels. For a large, macroscopic number of channels this noise becomes negligible. Under the realistic biological conditions, however, it may play an important role [21].

\subsection{The Hodgkin-Huxley Model}

Our starting point is the well-established model of Hodgkin and Huxley 18. The membrane patch of area $S$ is considered as an electrical capacitor possessing the specific area capacitance $C$. The membrane separates two ionic bath solutions (which in vivo correspond to the interior and the exterior of the excitable cell) with different concentrations of the ions of different sorts, mainly potassium, $\mathrm{K}^{+}$, sodium, $\mathrm{Na}^{+}$, and chloride, $\mathrm{Cl}^{-}$ions. The macroscopic concentration differences are kept approximately constant. In the cell, this task is accomplished by the ATP-driven ionic pumps. Furthermore, the ionic baths are on the average electri- 
cally neutral. However, due to the different ionic concentrations on the opposite sides of the semi-permeable membrane, the membrane becomes charged. As a consequence, an equilibrium transmembrane electrical potential difference emerges. The lipid membrane creates an almost impenetrable barrier for the ions. However, they can flow across the membrane through special ion selective pores created by specialized membrane proteins - the ion channels 22. The specific potassium, $I_{K}$, and sodium, $I_{N a}$, ion currents through the open ion channels are approximately proportional to the differences of the transmembrane potential $V$ and the specific (for the particular sort of ions) equilibrium potentials, $E_{K}$ and $E_{N a}$, respectively. The stochastically averaged, mean conductances, $G_{\mathrm{Na}}(m, h)$ and $G_{\mathrm{K}}(n)$, are, however, strongly nonlinear functions of $V$. This nonlinearity emerges due to the gating dynamics (see below). There exists also the leakage current $I_{L}$, mainly due to the chloride ions. If the membrane is driven by the external current $I_{\text {ext }}(t)$, the sum of the specific ion currents and the capacitive current, $I_{C}$, must be equal to $I_{e x t}(t)$ as a consequence of the charge conservation. Therefore, the equation for the transmembrane potential $V(t)$ reads

$$
\begin{aligned}
C \frac{d}{d t} V+G_{\mathrm{K}}(n)\left(V-E_{\mathrm{K}}\right)+G_{\mathrm{Na}}(m, h)(V & \left.-E_{\mathrm{Na}}\right) \\
& +G_{\mathrm{L}}\left(V-E_{L}\right)=I_{\mathrm{ext}}(t) .
\end{aligned}
$$

For a squid giant axon, the parameters in eq. (12.1) are $E_{\mathrm{N} a}=50 \mathrm{mV}$, $E_{\mathrm{K}}=-77 \mathrm{mV}, E_{L}=-54.4 \mathrm{mV}$, and $C=1 \mu \mathrm{F} / \mathrm{cm}^{2}$. Furthermore, the leakage conductance is assumed to be constant, $G_{\mathrm{L}}=0.3 \mathrm{mS} / \mathrm{cm}^{2}$. On the contrary, the sodium and potassium conductances are controlled by the voltage-dependent gating dynamics of single ion channels and are proportional to their respective numbers. These latter assumptions have been fully confirmed in the single-channel recordings by Neher, Sakmann and colleagues which indeed have proven that ion channels undergo the opening-closing stochastic gating dynamics [23]. In the Hodgkin-Huxley model, the opening of the potassium ion channel is governed by four identical activation gates characterized by the opening probability $n$. The channel is open when all four gates are open. In the case of sodium channel, the dynamics is governed by the three independent, identical fast activation gates $(m)$ and an additional slow, so-termed inactivation gate $(h)$. The independence of the gates implies that the probability $P_{\mathrm{K}, \mathrm{Na}}$ of the occurrence of the conducting conformation is $P_{\mathrm{K}}=n^{4}$ for a potassium channel and $P_{\mathrm{N} a}=m^{3} h$ for a sodium channel, respectively. In the mean-field description, the macroscopic potassium and sodium conductances thus read:

$$
G_{\mathrm{K}}(n)=g_{\mathrm{K}}^{\max } n^{4}, \quad G_{\mathrm{Na}}(m, h)=g_{\mathrm{Na}}^{\max } m^{3} h,
$$

where $g_{\mathrm{K}}^{\max }=36 \mathrm{mS} / \mathrm{cm}^{2}$ and $g_{\mathrm{N} a}^{\max }=120 \mathrm{mS} / \mathrm{cm}^{2}$ denote the maximal conductances (when all channels are open). The two-state, open-closing dynamics of the gates is given by the voltage dependent opening and closing rates $\alpha_{x}(V)$ and $\beta_{x}(V)(x=m, h, n)$, i.e. 


$$
\begin{aligned}
\alpha_{m}(V) & =\frac{0.1(V+40)}{1-\exp [-(V+40) / 10]}, \\
\beta_{m}(V) & =4 \exp [-(V+65) / 18], \\
\alpha_{h}(V) & =0.07 \exp [-(V+65) / 20], \\
\beta_{h}(V) & =\{1+\exp [-(V+35) / 10]\}^{-1}, \\
\alpha_{n}(V) & =\frac{0.01(V+55)}{1-\exp [-(V+55) / 10]}, \\
\beta_{n}(V) & =0.125 \exp [-(V+65) / 80] .
\end{aligned}
$$

Hence, the dynamics of the opening probabilities for the gates are given by:

$$
\dot{x}=\alpha_{x}(V)(1-x)-\beta_{x}(V) x, \quad x=m, h, n .
$$

The voltage equation (12.1), (12.2) and the rate equations of the gating dynamics (12.3), (12.4) define the original, purely deterministic Hodgkin-Huxley model [18] for the squid giant axon.

The rate constants in (12.3) are given in $m s^{-1}$ and the voltage in $m V$. These nonlinear Hodgkin-Huxley equations (12.1) (12.3) present a cornerstone model in neurophysiology. Within the same line of reasoning this model can be generalized to a mixture of different ion channels with various gating properties [24, 25].

The dynamics of the Hodgkin-Huxley model exhibits a complex, rich behavior which sensitively depends on the model parameters. For the squid giant axon parameters, the corresponding dynamics possesses a single fixed point and therefore does not exhibit a spiking activity in the absence of external stimulus, $I_{\text {ext }}(t)=0$. However, if a constant stimulus, $I_{\text {ext }}(t)=I_{0}$, is applied, the fixed point loses its stability with increasing strength $I_{0}$ upon $I_{0} \geq I_{1} \approx 9.763 \mu \mathrm{A} / \mathrm{cm}^{2}$. For such a super-threshold current strength, the membrane exhibits a periodic spiking activity which reflects the presence of a stable limit cycle, see Fig. 12.17. Upon decreasing the driving current strength, the spiking dynamics still persists below the threshold for excitation, i.e. also for $I<I_{1}$, until the diminishing current reaches the sub-critical value $I_{2} \approx 6.26 \mu \mathrm{A} / \mathrm{cm}^{2}$. Below this value, the limit cycle loses stability and the spiking activity vanishes. In conclusion, for $I_{2}<I_{0}<I_{1}$ both the stable fixed point and the stable limit cycle can indeed coexist. This feature is thus responsible for the hysteresis behavior in the spiking behavior versus the varying driving current strength, cf. Fig. 12.1 a.

Next we focus on a periodic sinusoidal driving,

$$
I_{\text {ext }}(t)=A \sin (\Omega t)
$$

with amplitude strength $A$ and angular driving frequency $\Omega$, see also in [26]. The corresponding dynamics becomes now even richer. In this case of periodic driving, the firing threshold $A_{t h}$ becomes frequency-dependent. The corresponding complexity for the phase diagram is depicted in Fig. 12.1b. In Fig. 12.1b, the border line $A_{t h}(\Omega)$ which separates the regime of no spiking from the regime 

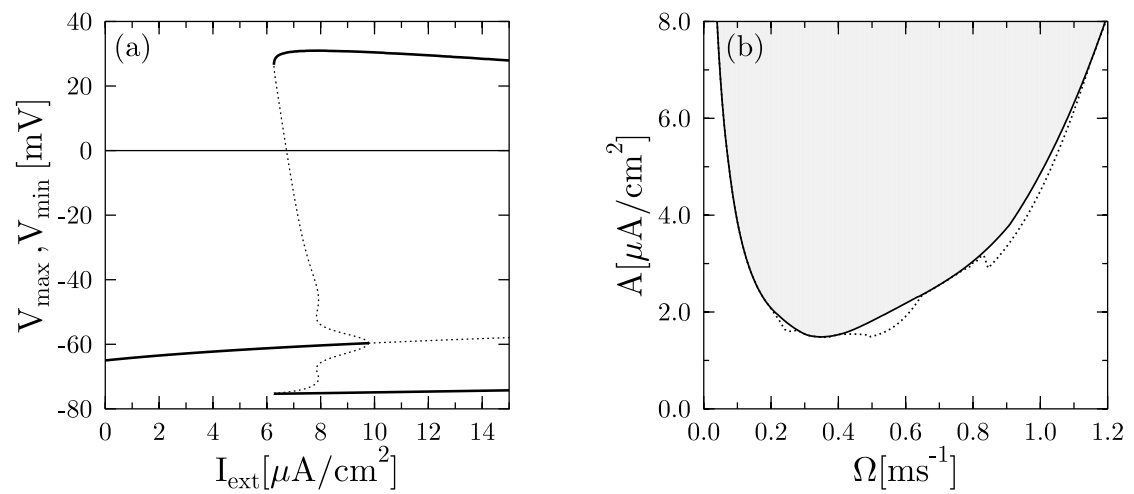

Fig. 12.1. The bifurcation diagram for the emergence of spiking behavior for the deterministic Hodgkin-Huxley model. The equilibrium voltage (fixed point) and the minimal and maximal voltage amplitudes, respectively, of the limit cycle oscillations are plotted in part a against the constant driving current strength. There exists a hysteretic behavior for the range $6.26<I_{\text {ext }}<9.763 \mu \mathrm{A} / \mathrm{cm}^{2}$ where the stable limit cycle exhibiting firing events and the stable fixed point coexist. $\mathbf{b}$ In the regime of periodic sinusoidal driving the situation is rather complex as well: a phase diagram is plotted as a function of the driving frequency $\Omega$ and the corresponding threshold amplitude $A_{t h}$ for firing. The solid line separates the phase with firing events (grey region) from the phase without spike occurrences (white region) for the case when the amplitude is correspondingly increased from zero. Upon starting from the regime with spiking the dotted line gives the phase separation line when the transition into the no-firing regime occurs. This reflects a typical hysteretic character

with an assured spiking behavior exhibits a hysteresis-like character. The solid line in Fig. 12.1b marks the transition to spiking when the driving strength is successively increased from zero driving strength. The occurrence of a minimum in this figure is quite remarkable: It implies that the system possesses an internal resonance frequency. This feature can be used for signal processing [20, 26].

\subsection{Stochastic Version of the Hodgkin-Huxley Model}

It has been suspected since the time of Hodgkin and Huxley, and known with certainty since the first single-channel recordings of Neher, Sakmann and colleagues, that voltage-gated ion channels are stochastic devices [23]. An essential drawback of the Hodgkin-Huxley model, however, is that it operates with the average number of open channels, thereby disregarding the corresponding number fluctuations (or, the so-called channel noise [23, 21]). These fluctuations, i.e. their strength, scale inversely proportional to the number of ion channels, see below. Thus, the original Hodgkin-Huxley model can be valid, strictly speaking, only within the limit of very large system size. We emphasize, however, that the size of an excitable membrane patch within a neuron is typically finite. 


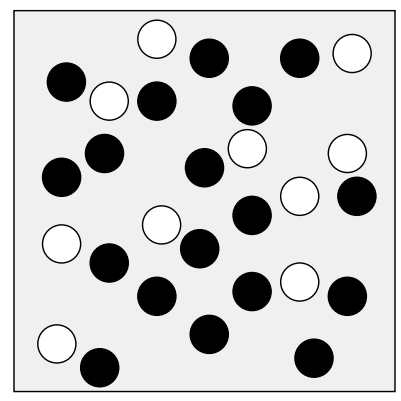

Fig. 12.2. Sketch of a membrane patch with potassium (white filled circles) and sodium (black filled circles) ion channels. The grey background indicates the leakage caused by additional nonvoltage dependent channels. The ion channels interact only globally, through the membrane voltage

In a small spherical neuron about $10 \mu \mathrm{m}$ in diameter, the membrane area is about $300 \mu \mathrm{m}^{2}$. Therefore, for a modest channel density of $\rho=30 \mu \mathrm{m}^{-2}$ there should be about $N=9000$ ion channels. The ratio of the standard deviation to the mean conductance of the whole ensemble of identical channels, which is $\delta G=$ $\sqrt{\left(1-P_{o}\right) /\left(P_{o} N\right)}$, where $P_{o}$ the stationary opening probability of a channel (see, e.g., in 21]), scales as $\delta G \propto 1 / \sqrt{N}$ with the number $N$ of ion channels. Therefore, the conductance fluctuations may become appreciable for a small cell. These fluctuations can also play a functional role 21. Besides, the spatial distribution of channels in receptor cell membranes is highly inhomogeneous and occurs in the form of clusters (see, e.g., an example in [27]) which are electrically coupled through the electrically passive pieces of membrane. As a consequence, the role of internal fluctuations cannot be a priori neglected [21]. As a matter of fact, as shown below, they can play a key role for SR in realistically small isolated clusters of ion channels like in Fig. 12.2

\subsubsection{Quantifying Channel Noise}

The role of channel noise for the neuron firing has been first studied by Lecar and Nossal as early as in 1971 [28]. The corresponding stochastic generalizations of Hodgkin-Huxley model (within a kinetic model which corresponds to the above given description) has been put forward by DeFelice et al. 29] and others; see [21] for a review and further references therein. The main conclusion of these previous studies is that the channel noise can be functionally important for neuron dynamics. In particular, it has been demonstrated that channel noise alone can give rise to a spiking activity even in the absence of any stimulus [21, 29, 30, see also in Fig. 12.3.

To include the channel noise influence in a theoretical modeling within the stochastic kinetic schemes [21, 29], however, necessitates extensive numerical simulations 31. To aim at a less cumbersome numerical scheme we use a short-cut procedure that starts from (12.4) in order to derive a corresponding set of Langevin equations for a stochastic generalization of the Hodgkin-Huxley equations of 


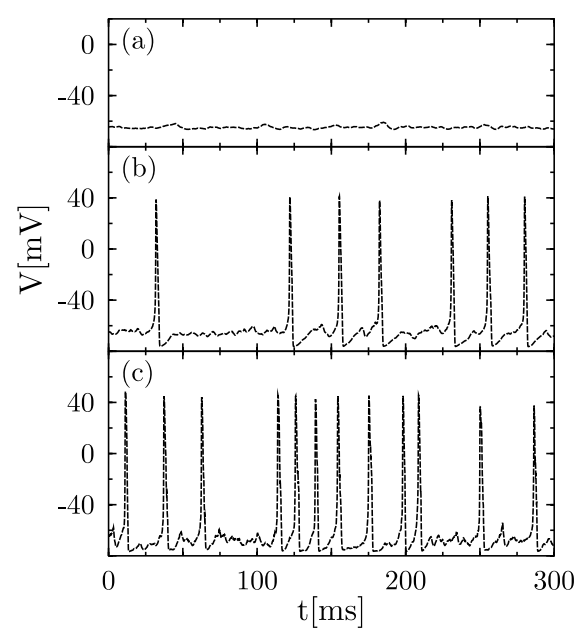

Fig. 12.3. Numerical simulation of the stochastic Hodgkin-Huxley system (12.1), (12.6), (12.7) with vanishing external stimulus. We computed several realizations of the voltage signal for different numbers of the ion channels: a $N_{\mathrm{N} a}=6000, N_{\mathrm{K}}=1800 ; \mathbf{b} N_{\mathrm{N} a}=$ $600, N_{\mathrm{K}}=180 ;$ and $\mathbf{c} N_{\mathrm{N} a}=60$, $N_{\mathrm{K}}=18$. Upon decreasing the system size the influence of channel noise on the spontaneous firing dynamics becomes more and more pronounced. Note that the non-stochastic Hodgkin-Huxley model does not exhibit spikes at all for the parameters given in the text and in the absence of external stimuli

the type put forward by Fox and Lu [32]. Following their reasoning we substitute the equations (12.4) with the corresponding Langevin generalization:

$$
\begin{aligned}
\dot{m} & =\alpha_{m}(V)(1-m)-\beta_{m}(V) m+\xi_{m}(t), \\
\dot{h} & =\alpha_{h}(V)(1-h)-\beta_{h}(V) h+\xi_{h}(t), \\
\dot{n} & =\alpha_{n}(V)(1-n)-\beta_{n}(V) n+\xi_{n}(t),
\end{aligned}
$$

with independent Gaussian white noise sources of vanishing mean. The noise autocorrelation functions depend on the stochastic voltage and the corresponding total number of ion channels as follows:

$$
\begin{aligned}
\left\langle\xi_{m}(t) \xi_{m}\left(t^{\prime}\right)\right\rangle & =\frac{2}{N_{\mathrm{Na}}} \frac{\alpha_{m} \beta_{m}}{\left(\alpha_{m}+\beta_{m}\right)} \delta\left(t-t^{\prime}\right), \\
\left\langle\xi_{h}(t) \xi_{h}\left(t^{\prime}\right)\right\rangle & =\frac{2}{N_{\mathrm{Na}}} \frac{\alpha_{h} \beta_{h}}{\left(\alpha_{h}+\beta_{h}\right)} \delta\left(t-t^{\prime}\right), \\
\left\langle\xi_{n}(t) \xi_{n}\left(t^{\prime}\right)\right\rangle & =\frac{2}{N_{\mathrm{K}}} \frac{\alpha_{n} \beta_{n}}{\left(\alpha_{n}+\beta_{n}\right)} \delta\left(t-t^{\prime}\right) .
\end{aligned}
$$

In order to confine the conductances between the physically allowed values between 0 (all channels are closed) and $g^{\max }$ (all channels are open) we have implemented numerically the constraint of reflecting boundaries so that $m(t), h(t)$ and $n(t)$ are always located between zero and one [32].

Moreover, the numbers $N_{\mathrm{Na}}$ and $N_{\mathrm{K}}$ depend on the actual area $S$ of the membrane patch. With the assumption of homogeneous ion channels densities, $\rho_{\mathrm{Na}}=60 \mu \mathrm{m}^{-2}$ and $\rho_{\mathrm{K}}=18 \mu \mathrm{m}^{-2}$, the following scaling behavior follows:

$$
N_{\mathrm{Na}}=\rho_{\mathrm{Na}} S, \quad N_{\mathrm{K}}=\rho_{\mathrm{K}} S .
$$


Upon decreasing the system size $S$, the fluctuations and, hence, the internal noise increases. Consequently, with abating cell membrane patch the spiking behavior changes dramatically, cf. Fig. 12.3

The numerical integration is carried out by the standard Euler algorithm with the step size $\Delta t \approx 2 \cdot 10^{-3} \mathrm{~ms}$. The "Numerical Recipes" routine ran2 is used for the generation of independent random numbers [33] with the BoxMuller algorithm providing the Gaussian distributed random numbers. The total integration time is chosen to be a multiple of the driving period $T_{\Omega}=2 \pi / \Omega$, as to ensure that the spectral line of the driving signal is centered on a computed value of the power spectral densities. From the stochastic voltage signal $V(t)$ we extract a point process of spike occurrences $\left\{t_{i}\right\}$ :

$$
u(t):=\sum_{i=1}^{N} \delta\left(t-t_{i}\right),
$$

where $N$ is the total number of spikes occurring during the elapsed time interval. The occurrence of a spike in the voltage signal $V(t)$ is detected by upwardcrossing a certain detection threshold value $V_{0}$. Obviously, the threshold can be varied over a wide range with no effect on the resulting spike train dynamics.

The power spectral density of the spike train $\left(\mathrm{PSD}_{u}\right)$ has been analyzed in the absence and in the presence of periodic stimulus and noise. In order to quantify $\mathrm{SR}$, we obtain from the $\mathrm{PSD}_{u}$ the spectral power of the transmitted periodic signal, $\eta$, as the difference between the peak value of the spectral line and its background offset located at the driving frequency $\Omega$. The another important measure, signal-to-noise ratio (SNR), is then given by the ratio of the spectral power of signal to the background offset (in the units of spectral resolution of signals).

\subsubsection{Stochastic Resonance}

First, we focus our attention on SR in absence of external noise, see Fig. 12.4 and Fig. 12.5 a. Here, we discover the novel effect of genuine intrinsic stochastic resonance, where the response of the system to the sub-threshold external stimulus is optimized solely due to internal, ubiquitous noise. For the given parameters, $\mathrm{SR}$ in the spectral amplification of signal occurs at $S \approx 10 \mu \mathrm{m}^{2}$ and in the signal-to-noise ratio at a different value $S \approx 32 \mu \mathrm{m}^{2}$. Starting from $S \approx 10$ $\mu \mathrm{m}^{2}$, growing internal noise monotonically deteriorates the amplitude of system response at the signal frequency. Moreover, upon reaching $S \approx 32 \mu \mathrm{m}^{2}$ it deteriorates the quality of signal transduction which is measured by SNR. In this respect, it is worth mentioning that SNR also measures in effect the rate of information transfer [34], but for small-amplitude signals only [35].

Under such circumstances, one would predict that the addition of an external noise (which corresponds to the conventional situation in biological SR studies) cannot improve $\eta$ and SNR further, i.e. conventional SR will not be exhibited. In order to verify this prediction, we contaminated the periodic stimulus (12.5) 

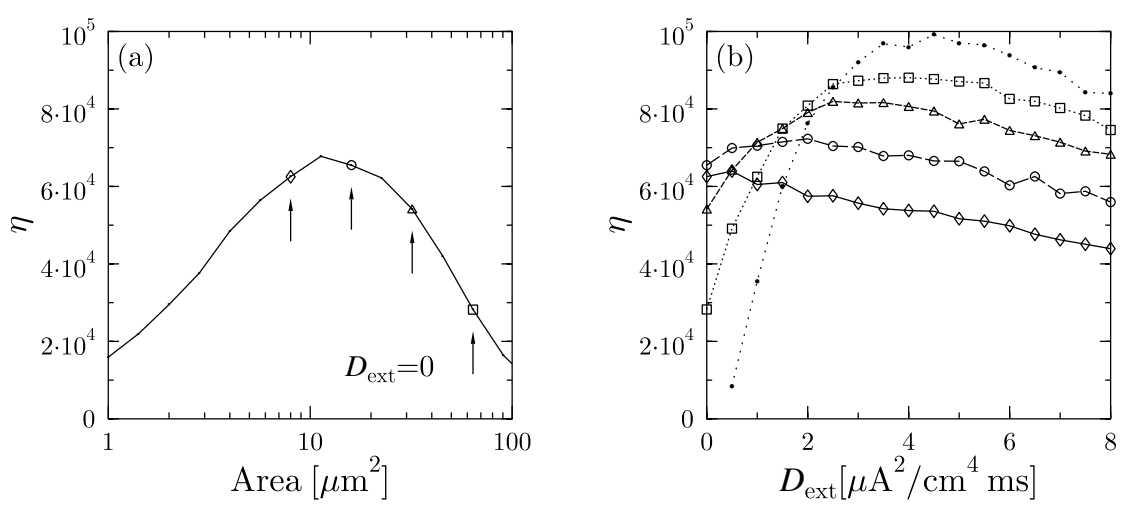

Fig. 12.4. The spectral amplification $\eta$ of an external sinusoidal stimulus with amplitude $A=1.0 \mu \mathrm{A} / \mathrm{cm}^{2}$ and angular frequency $\Omega=0.3 \mathrm{~ms}^{-1}$ for different observation areas: a no external noise is applied; $\mathbf{b}$ SNR versus the external noise for the system sizes indicated by the arrows in Fig. 12.5 a: $S=8 \mu \mathrm{m}^{2}$, solid line through the diamonds; $S=16 \mu \mathrm{m}^{2}$, long dashed line connecting the circles; $S=32 \mu \mathrm{m}^{2}$, short dashed line through the triangles; $S=64 \mu^{2}$, dotted line connecting the squares. The situation with no internal noise (i.e., formally $S \rightarrow \infty$ ) is depicted by the dotted line connecting the filled dots
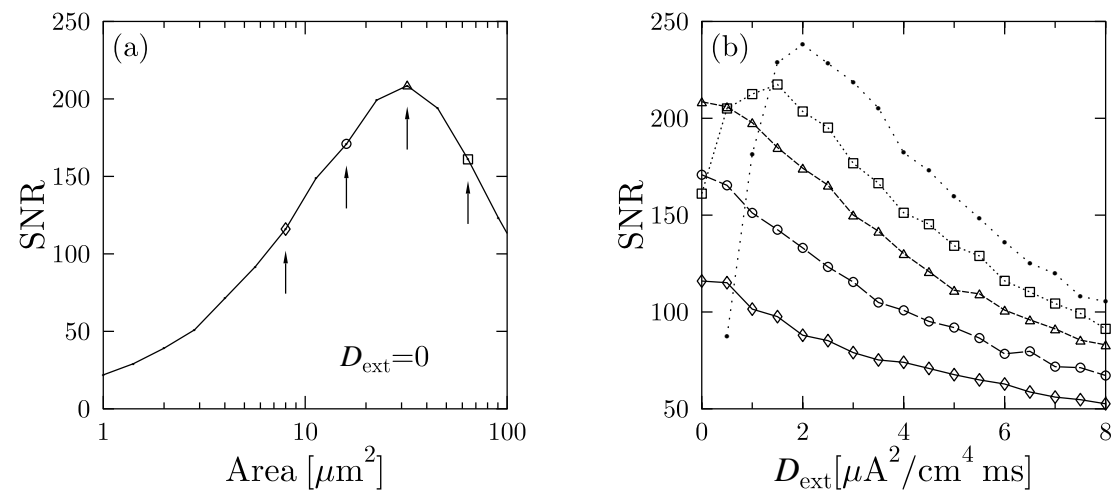

Fig. 12.5. The signal-to-noise ratio (SNR) for the same parameters as in Fig. 12.4

by the addition of Gaussian white noise $\zeta(t)$. The latter one possesses the autocorrelation function

$$
\left\langle\zeta(t) \zeta\left(t^{\prime}\right)\right\rangle=2 D_{\mathrm{ext}} \delta\left(t-t^{\prime}\right)
$$

and the noise strength $D_{\text {ext }}$. The corresponding results, depicted in Fig. 12.4b and Fig. 12.5b, fully confirm the above prediction. Conventional stochastic resonance therefore occurs only for large membrane patches beyond optimal sizes and reaches saturation in the limit $S \rightarrow \infty$ (limit of the deterministic HodgkinHuxley model). Thus, the observed biological SR [10, 11] is rooted in the collec- 

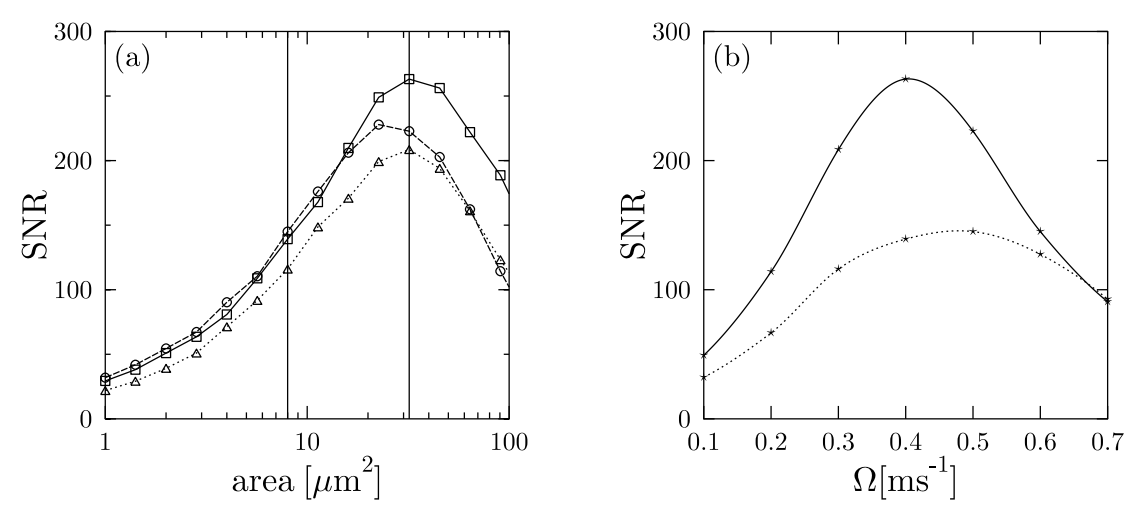

Fig. 12.6. The signal-to-noise ratio (SNR) for a sub-threshold external stimulus with amplitude $A=1.0 \mu \mathrm{A} / \mathrm{cm}^{2}$ and different angular frequencies: a SNR versus the observation area for $\Omega=0.3 \mathrm{~ms}^{-1}$ (dotted line through the triangles), $\Omega=0.4 \mathrm{~ms}^{-1}$ (solid line connecting the squares), and $\Omega=0.5 \mathrm{~ms}^{-1}$ (dashed line through the circles); b SNR versus the driving frequency for two areas $\left(S=8 \mu \mathrm{m}^{2}\right.$, dotted line; $S=32 \mu \mathrm{m}^{2}$, solid line), depicted by vertical lines in Fig. 12.6a. The curves exhibit clear maxima and, therefore, a combination of the stochastic resonance with the conventional frequency resonance takes place

tive properties of large ion channels arrays, where ion channels are globally coupled via the common membrane potential $V(t)$.

In addition, by changing the driving frequency we rediscover the effect of combined stochastic resonance and conventional resonance [20, 26, cf. Fig. 12.6] In other words, SNR becomes optimized not only versus the patch size, but also versus the driving frequency. Moreover, due to the noisy character of gating variables, the mean frequency of a corresponding random limit cycle in the stochastic Hodgkin-Huxley model (12.1),(12.6),(12.7) depends on the membrane patch area. Thus, the maxima of SNR are located for various system sizes at different driving frequencies. This effect may be used for frequency tuning in the biological signal transduction.

\subsection{Conclusions}

In conclusion, we have investigated the stochastic resonance in a noisy generalization of the Hodgkin-Huxley model. The spontaneous fluctuations of the membrane conductivity due to the individual ion channel dynamics has systematically been taken into account. We have shown that the excitable membrane patches exhibit a spontaneous spiking activity due to the omnipresent internal noise.

The main result of this study refers to the phenomenon of intrinsic SR. Here, the channel noise alone gives rise to SR behavior, cf. Fig. 12.4 a and Fig. 12.5, 
(see also 31]). Moreover, such intrinsic SR becomes optimized versus the driving angular frequency, cf. Fig. 12.6. Conventional SR versus the external noise intensity also takes place, but for sufficiently large membrane patches, where the internal noise strength alone is not yet at its optimal value. We thus conclude that the observed biological SR likely is rooted in the collective properties of globally coupled ion channel assemblies.

The authors gratefully acknowledge support for this work by the Deutsche Forschungsgemeischaft, SFB 486 Manipulation of matter on the nanoscale, project $\mathrm{A} 10$.

\section{References}

1. D.J. Watts and S.H. Strogatz, Nature (London) 393, 440-442 (1998)

2. A.L. Barabasi and R. Albert, Science, 286, 509-512 (1999)

3. R. Albert and A.L. Barabasi, Rev. Mod. Phys. 74, 47-98 (2002)

4. S.N. Dorogovtsev and J.F.F. Mendes, Adv. Phys. 51, 1079-1187 (2002)

5. R. Cohen, K. Erez, D. ben-Avraham and S. Havlin, Rev. Phys. Lett. 85, 4626-4628 (2000)

6. R. Cohen, K. Erez, D. ben-Avraham and S. Havlin, Rev. Phys. Lett. 86, 3682-3685 (2001)

7. R. Cohen, D. ben-Avraham and S. Havlin, Rev. Rev. E. 66, 036113 (2002)

8. L. Gammaitoni, P. Hänggi, P. Jung, and F. Marchesoni, Rev. Mod. Phys. 70, 223-288 (1998)

9. V.S. Anishchenko, A.B. Neiman, F. Moss and L. Schimansky-Geier, Usp.Fiz. Nauk 169, 7-38 (1999) [Physics - Uspekhi 42, 7-36 (1999)]

10. J.K. Douglass, L. Wilkens, E. Pantazelou, and F. Moss, Nature (London) 365, 337-340 (1993)

11. J.E. Levin, and J.P. Miller, Nature (London) 380, 165-168 (1996)

12. J.J. Collins, T.T. Imhoff, and P. Grigg, Nature (London) 383, 770 (1996)

13. E. Simonotto, M. Riani, C. Seife, M. Roberts, J. Twitty, and F. Moss, Phys. Rev. Lett. 78, 1186-1189 (1997)

14. I. Hidaka, D. Nozaki, and Y. Yamamoto, Phys. Rev. Lett. 85, 3740-3743 (2000)

15. S.M. Bezrukov, and I. Vodyanoy, Nature (London) 378, 362-364 (1995); 385, 319$321(1997)$

16. I. Goychuk, and P. Hänggi, Phys. Rev. E 61, 4272-4280 (2000)

17. D. Petracchi, M. Pellegrini, M. Pellegrino, M. Barbi, and F. Moss, Biophys. J. 66, 1844-1852 (1994)

18. A.L. Hodgkin, and A.F. Huxley, J. Physiol. (London) 117, 500-544 (1952)

19. A. Longtin, J. Stat. Phys. 70, 309-327(1993);

K. Wiesenfeld, D. Pierson, E. Pantazelou, C. Dames, and F. Moss, Phys. Rev. Lett. 72, 2125-2129 (1994);

J.J. Collins, C.C. Chow, A.C. Capela, and T.T. Imhoff, Phys. Rev. E 54, 55755584 (1996);

S.-G. Lee, and S. Kim, Phys. Rev. E 60, 826-830 (1999)

20. B. Lindner, and L. Schimansky-Geier, Phys. Rev. E 61, 6103-6110 (2000)

21. J.A. White, J.T. Rubinstein, and A.R. Kay, Trends Neurosci. 23, 131-137 (2000) 
22. B. Hille, Ionic Channels of Excitable Membranes, 2nd ed. (Sinauer Associates, Sunderland, MA 1992)

23. B. Sakmann, and E. Neher, Single-Channel recording (Plenum Press, 1995)

24. R.J. Nossal, and H. Lecar, Molecular and Cell Biophysics (Addison-Wesley, Redwood City 1991)

25. S.B. Lowen, L.S. Liebovitch, and J.A. White, Phys. Rev. E 59, 5970-5980 (1999)

26. Y. Yu, W. Wang, J. Wang, and F. Liu, Phys. Rev. E 63, 021907 (2001)

27. N.P. Issa and A.J. Hudspeth, Proc. Natl. Acad. Sci. USA 91, 7578-7582 (2001)

28. H. Lecar, and R. Nossal, Biophys. J. 11, 1068-1084 (1971)

29. J.R. Clay, and L.J. DeFelice, Biophys. J. 42, 151-157 (1983);

A.F. Strassberg, and L.J. DeFelice, Neural Comput. 5, 843-855 (1993);

L.J. DeFelice, and A. Isaac: Chaotic states in a random world, J. Stat. Phys. 70, 339-354 (1993)

30. G. Schmid, and I. Goychuk, P. Hänggi, Europhys. Lett. 56, 22-28 (2001)

31. P. Jung, and J.W. Shuai, Europhys. Lett. 56, 29-35 (2001)

32. R.F. Fox R, and Y. Lu, Phys. Rev. E 49, 3421-3431 (1994)

33. W.H. Press, S.A. Teukolsky, W.T. Vetterling, and B.P. Flannery, Numerical Recipes in $C$, 2nd ed. (Cambridge Univ. Press, Cambridge 1992)

34. F. Rieke, D. Warland, R. de Ruyter van Steveninck, and W. Bialek, Spikes: Exploring the Neural Code (MIT Press, Cambridge, MA, 1997)

35. I. Goychuk, Phys. Rev. E 64, 021909 (2001) 